\title{
Collision Occurrence of Sinonasal Carcinosarcoma and Pituitary Adenoma: A Case Report and Literature Review.
}

\section{Yufei Liu}

The First Affiliated Hospital of Shenzhen University Jihu Yang

The First Affiliated Hospital of Shenzhen University

\section{Xiejun Zhang}

The First Affiliated Hospital of Shenzhen University

\section{Yanhua Sun}

The First Affiliated Hospital of Shenzhen University

\section{Fanfan Chen}

The First Affiliated Hospital of Shenzhen University

\section{Nan Ji}

Beijing Tiantan Hospital, Capital Medical University

\section{Liwei Zhang}

Beijing Tiantan Hospital, Capital Medical University

Guodong Huang ( $\sim$ jxgd211@163.com )

Shenzhen Second People's Hospital https://orcid.org/0000-0002-2848-7581

\section{Case report}

Keywords: Sinonasal malignancy, Pituitary adenoma, Endoscopic, Expanded endonasal approach

Posted Date: December 23rd, 2020

DOI: https://doi.org/10.21203/rs.3.rs-124932/v1

License: (c) (1) This work is licensed under a Creative Commons Attribution 4.0 International License. Read Full License 


\section{Abstract}

BACKGROUND: Collision occurrences of sinonasal carcinosarcoma and pituitary adenoma are rarely reported. Sinonasal carcinosarcomas represent rare neoplasms with invasive characteristics and unfavorable prognoses.

CASE DESCRIPTION: We present a rare case of a collision occurrence of sinonasal carcinosarcoma and pituitary adenoma in a 45-year-old male patient. MRI demonstrated a large mass involving the sellar region, nasal cavity, paranasal sinuses and anterior cranial base. Total surgical resection with a pure endoscopic expanded endonasal approach was performed successfully with neuronavigational assistance. Histopathologic results were a carcinosarcoma in the nasal cavity, paranasal sinuses, and anterior cranial base and a pituitary adenoma in the intrasellar zone. The Ki-67 index of the carcinosarcoma was high (more than 95\%). Although the patient received chemotherapy, he died 6 months after surgery because of in situ recurrence and extensive metastatic growth.

CONCLUSIONS: Collision occurrences of sinonasal carcinosarcoma and pituitary adenoma are rare events. Such tumor could be removed successfully by neuronavigational guidance with a pure endoscopic expanded endonasal approach. A contralateral nasal septum mucosa flap without tumor invasion can be used as a kind of skull base repair material. A high Ki-67 index may be a biomarker of rapid tumor progression and poor prognosis in such patients.

\section{Introduction}

Sinonasal malignancies represent rare but aggressive tumors that are difficult to treat. The incidence of sinonasal malignancies is 0.83 per 100,000 people, with a slight male predominance ${ }^{[1]}$. Symptoms tend to occur secondary to the mass effect on surrounding tissue; these may include nasal obstruction, facial pain, headache, rhinorrhea, bleeding, and anosmia. Sinonasal malignancies include a varied group of histopathologic entities, such as squamous cell neoplasms, adenoid cystadenocarcinomas, olfactory neuroblastoma, neuroepitheliomatous neoplasms, mature B-cell non-Hodgkin's lymphoma, nevi and melanomas, and adenocarcinomas ${ }^{[1-2]}$. Therapeutic strategies include surgery, radiotherapy, and chemotherapy. Endonasal endoscopic resection of sinonasal malignancies plays a crucial part in the multidisciplinary management of patients with these complex lesions ${ }^{[3]}$. We herein present a patient with a rare collision occurrence of sinonasal carcinosarcoma and pituitary adenoma who underwent a pure endoscopic expanded endonasal approach to remove these tumors.

\section{Case Description}

The patient, a 45-year-old male, was admitted to the hospital complaining of "anosmia for 2 months". Past medical history, family history and social history were not significant. Apart from anosmia, he had no other symptoms or neurological dysfunction. Computed tomography (Fig. 1A-B) and MRI demonstrated an extensive mass that had not only filled the right and left side of the nasal cavity and 
paranasal sinuses but also eroded the skull base, causing right frontal lobe compression. The lesion was T1WI isodense, and slightly T2WI hyperintense, with irregular margins and strong uptake of Gd-DTPA (Fig. 1C-E). In addition, a mass lesion was observed in the sellar region, obscuring a clear view of a normal pituitary. The size of the sellar mass was approximately $20 \times 18 \times 18 \mathrm{~mm}^{3}$. The serum ACTH value was $59.6 \mathrm{pg} / \mathrm{ml}$ (reference value: $0-46 \mathrm{pg} / \mathrm{ml}$ ), and the levels of other serum hormones were within normal ranges.

The decision was made to perform a single resection of the two lesions through a pure endoscopic expanded endonasal approach. The tumor and eroded middle turbinate in the right side of the nasal cavity was initially resected (Fig. 2A). Because the right mucoperiosteal flap of the nasal septum was eroded, the left mucoperiosteal flap of the nasal septum and inferior nasal meatus was obtained for skull base reconstruction (Fig. 2B). After resection of part of the tumor and the anterior wall of the sphenoid sinus, the thin seller floor was visualized. Then, the seller floor was removed, and the seller dura was cut and moved. A soft, red and white tumor (Fig. 2C) could be completely removed from the intrasellar region by curettage without cerebrospinal fluid fistula. Seller floor reconstruction in this case included dural suture with 4 - 0 thread (Fig. 2D), return of the bone of the sellar floor and the application of artificial dura mater. Neuronavigational guidance was used because of its ability to accurately determine location (Fig. 1F), helping the operator resect the tumor and the eroded ethmoid sinus and anterior cranial base (Fig. 2E-F). The fat and muscle fascia of the quadriceps were obtained from the right leg. Along with the left mucoperiosteal flap of the nasal septum and inferior nasal meatus, the harvested leg tissues were used for anterior skull base reconstruction (Fig. 2H). The postoperative course was uneventful. MRI reexamination revealed the postoperative pathological results of the gross resection. Histological examination showed a carcinosarcoma ( $90 \%$ of the mass was undifferentiated carcinoma with neuroendocrine characteristics, and $10 \%$ was rhabdomyosarcoma) in the nasal cavity and paranasal sinuses and a pituitary adenoma in the intrasellar zone. The immunohistochemical results (Fig. 3) from the tumor involving the nasal cavity, paranasal sinuses and anterior cranial base were CK (+), EMA (-), GFAP (-), Ki-67 (more than 95\%), NSE (slightly positive), S-100 (focally positive), Syn (focally positive), P63 (-), Olig - 2 (-), CD56 (+), CgA (focally positive), Des (focally positive), CK 8/18 (+), CK - L (+), CD99 (focally positive), Vim (focally positive), Myogenin (slightly positive), and MYOD1 (focally positive). The immunohistochemical results from the sellar tumor were ACTH (-), CAM5.2 (-), ER (focally positive), FSH (focally positive), GH (-), Ki-67 (< 1\% +), LH (-), PIT - 1 (+), PRL (-), SF-1 (focally positive), T - PIT (-), TSH $(-)$, reticular fiber staining (nesting pattern), CK (-), EMA (-), GFAP (-), NSE (focally positive), S-100 (-), Syn $(+)$, ABT (-), and Olig-2 (-). After discharge, the patient underwent chemotherapy (cisplatin and etoposide) in another hospital. Unfortunately, he died 6 months after surgery because of tumor recurrence and extensive metastasis.

\section{Discussion}

Sinonasal carcinosarcoma, with an incidence of 0.83 per 100,000 population, is a rare malignant tumor in adults and is associated with poor survival. Black race may be a risk factor for poorer disease-specific 
survival from these malignancies. Surgical removal followed by radiotherapy, chemotherapy or a combination of radiotherapy and chemotherapy is considered for many patients.

Collision occurrences of sinonasal carcinosarcoma and pituitary adenoma are rarely reported. The sinonasal carcinosarcoma in this case consisted of undifferentiated carcinoma with neuroendocrine characteristics and rhabdomyosarcoma. de Silva AC et al. reported a case of combined gonadotrophic pituitary tumor and rhabdomyosarcoma. They maintained two possibilities of the etiology of the rhabdomyosarcomatous component: metaplastic transformation from a pituitary adenoma or independent collision tumors ${ }^{[4]}$. Virginia E. et al. advocated a serious suspicion for coexistent rhabdomyosarcoma with pituitary adenoma in cases of atypical pituitary adenoma with a stromal respons $\mathrm{e}^{[5]}$. However, the patient underwent adjuvant radiation, which may be a risk factor associated with rhabdomyosarcoma ${ }^{[5]}$. In the present case, the patient was diagnosed with a collision occurrence of sinonasal rhabdomyosarcoma and pituitary adenoma after the operation without a definite etiology. In addition, a sinonasal undifferentiated carcinoma as a third primary neoplasm was reported in an elderly patient with a history of pituitary prolactinoma and metastatic pancreatic carcinoma ${ }^{[6]}$. More patients with this collision occurrence are required to further assess the potential underlying mechanism.

Endonasal endoscopy plays a critical role in the surgical treatment of sinonasal malignancies, including those with skull base invasion ${ }^{[3,7,8]}$. The extended endoscopic endonasal approach can provide a wide, clear operative field in the skull base and the intrasellar and suprasellar zones. After thorough consultation, the decision was made for the present case to remove the large lesion through a pure endoscopic extended endonasal approach. The intrasellar tumor showed red and white fish-like tissue, which was removed easily by aspiration. However, the sinonasal tumor was composed of tissue with a tough texture that eroded the ethmoid sinus and anterior cranial base and adhered to the frontal lobe.

The next key step after tumor resection is to close CSF leaks and dural defects. There is a preference for autologous materials (such as nasoseptal flaps, fat, fascia lata, bone and mucoperiosteum taken from the middle turbinate) and collagen matrix ${ }^{[9-11]}$. After resection of the lesion in the saddle area, the intrasellar arachnoid was intact, but the right nasal septum had been invaded by the tumor, so we used a right nasal septum mucosal flap to repair the anterior skull base. The postoperative course was uneventful, lacking CSF leakage.

Ki-67 protein expression is strongly associated with the cell growth and proliferation of many kinds of tumor and is widely used in routine pathological examination as a proliferation marker. Recent research has suggested that Ki-67 may be a promising molecular candidate for the treatment of various malignancies ${ }^{[12-13]}$. The Ki-67 index of the carcinosarcoma in this case was more than $95 \%$. The patient died 6 months after surgery because of tumor in situ recurrence and extensive metastasis, which indicated that a high Ki-67 index may be a biomarker of rapid tumor progression and poor prognosis in such patients. More cases are needed to confirm this theory.

\section{Conclusion}


This was a rare case of a collision occurrence of sinonasal carcinosarcoma and pituitary adenoma. The extended endoscopic endonasal approach provides a wide, clear, multiple angle operative field in the skull base and the supersellar region. The endoscopic approach is safe and effective for selected anterior cranial base and sellar zone tumors. In addition to the materials commonly used to repair the skull base, such as fat, muscle and artificial dura, a contralateral nasal septum mucosa flap without tumor invasion can be used as a kind of skull base repair material. A high Ki-67 index may be a biomarker of rapid tumor progression and poor prognosis in such patients.

\section{Declarations}

Ethics approval and consent to participate: The patient underwent surgery with full informed consent and signed a consent form. The treatment was completely in line with medical ethics.

Consent for publication: Not applicable.

Availability of data and materials: All data generated or analysed during this study are included in this published article.

Competing interests: The authors report no conflict of interest.

Funding: This work was supported by the Science and Technology Planning Project of Guangdong Province (Grant no. 201700892).

Authors' contributions: $Y L, J Y$ and $X Z$ : writing the paper. YS: providing and modifying pathological images. FC, NJ, LZ and GH: correction of the paper. All authors have read and approved the manuscript.

Acknowledgments: We would like to thank the patient and his family who trusted us.

\section{References}

1. Dutta R, Dubal PM, Svider PF, et al. (2015) Sinonasal malignancies: a population-based analysis of site-specific incidence and survival. Laryngoscope 125:2491-7. doi: 10.1002/lary.25465.

2. Li J, Yuan H, Li Y, et al. (2019) Lin Chung Er Bi Yan Hou Tou Jing Wai Ke Za Zhi. 2019 33:11761180. doi:10.13201/j.issn.1001-1781.

3. Roxbury CR, Ishii M, Richmon JD, et al. (2016) Endonasal Endoscopic Surgery in the Management of Sinonasal and Anterior Skull Base Malignancies. Head Neck Pathol 10:13-22. doi:10.1007/s12105016-0687-8.

4. de Silva AC, Rodriguez FJ, Aldecoa I, et al. (2016) Compound gonadotrophic pituitary adenoma and rhabdomyosarcoma. Histopathology 68:1111-1114. doi:10.1111/his.12890.

5. Duncan VE, Nabors LB, Warren PP, et al. (2016) Primary Sellar Rhabdomyosarcoma Arising in Association With a Pituitary Adenoma. Int J Surg Pathol 24:753-756.

doi:10.1177/1066896916658955. 
6. Chi JJ, Feldman MD, Palmer JN. (2015) Sinonasal undifferentiated carcinoma as a third primary neoplasm: A case report and review of the literature. Ear Nose Throat J 94:178-182.

7. Volpi L, Bignami M, Lepera D, et al. (2019) Endoscopic endonasal resection of adenoid cystic carcinoma of the sinonasal tract and skull base. Laryngoscope 129:1071-1077. doi:10.1002/lary.27485.

8. Radulesco T, Penicaud M, Dessi P, et al. (2017) Endoscopic surgery for sinonasal tumors: The transcribriform approach. J Stomatol Oral Maxillofac Surg 118:248-250. doi:10.1016/j.jormas.2017.06.015.

9. Kassam A, Carrau RL, Snyderman CH, et al. (2005) Evolution of 200 reconstructive techniques following endoscopic expanded endonasal approaches. Neurosurg Focus 19:E8. 202.

10. Kassam AB, Thomas A, Carrau RL, et al. (2008) Endoscopic reconstruction of the cranial base using a pedicled nasoseptal flap. Neurosurgery 63(1 Suppl 1):ONS44-52; discussion ONS52-3. doi: 10.1227/01.neu.0000297074.13423.f5.

11. Sciarretta V, Mazzatenta D, Ciarpaglini R, et al. (2010) Surgical repair of persisting CSF leaks following standard or extended endoscopic transsphenoidal surgery for pituitary tumor. Minim Invasive Neurosurg 53:55-59. doi:10.1055/s-0029-1246161.

12. Yang C, Zhang J, Ding M, et al. (2018) Ki67 targeted strategies for cancer therapy. Clin Transl Oncol 20:570-575. doi:10.1007/s12094-017-1774-3.

13. Menon SS, Guruvayoorappan C, Sakthivel KM, et al. (2019) Ki-67 protein as a tumour proliferation marker. Clin Chim Acta 491:39-45. doi:10.1016/j.cca.

\section{Figures}
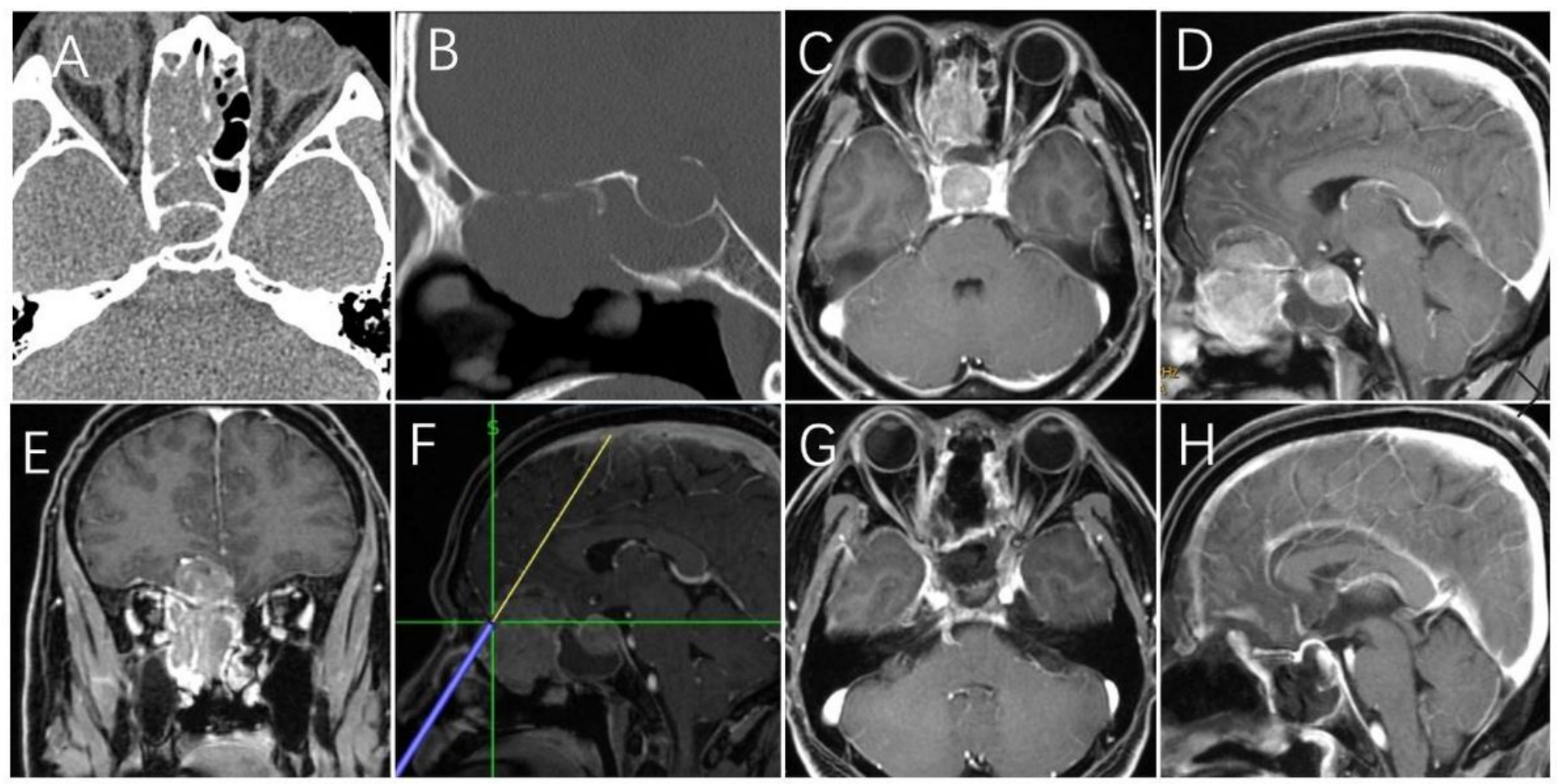


\section{Figure 1}

Computed tomography (Figure 1A-B) and MRI demonstrated an extensive mass that had not only filled the right and left side of the nasal cavity and paranasal sinuses but also eroded the skull base, causing right frontal lobe compression. The lesion was T1WI isodense, and slightly T2WI hyperintense, with irregular margins and strong uptake of Gd-DTPA (Figure 1C-E).

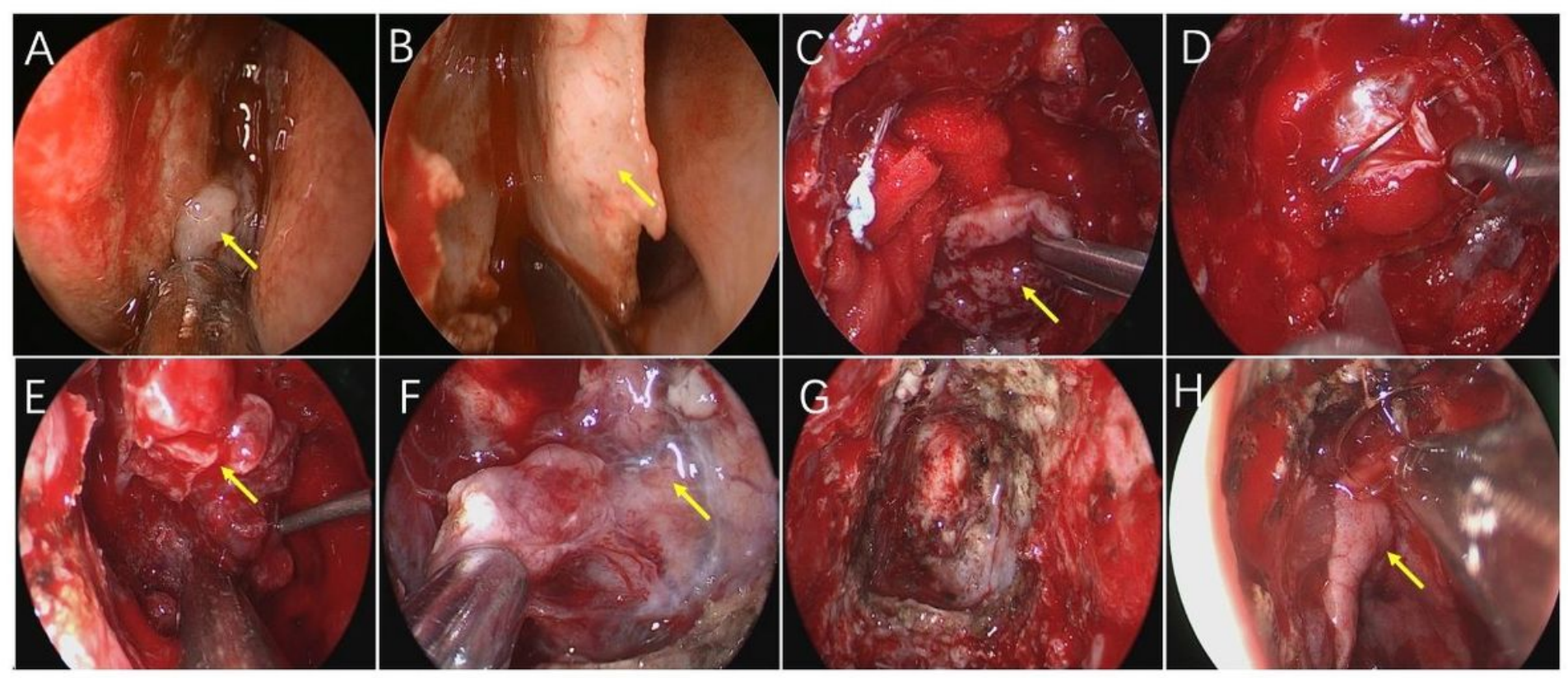

\section{Figure 2}

The tumor and eroded middle turbinate in the right side of the nasal cavity was initially resected (Figure $2 \mathrm{~A})$. Because the right mucoperiosteal flap of the nasal septum was eroded, the left mucoperiosteal flap of the nasal septum and inferior nasal meatus was obtained for skull base reconstruction (Figure 2B). After resection of part of the tumor and the anterior wall of the sphenoid sinus, the thin seller floor was visualized. Then, the seller floor was removed, and the seller dura was cut and moved. A soft, red and white tumor (Figure 2C) could be completely removed from the intrasellar region by curettage without cerebrospinal fluid fistula. Seller floor reconstruction in this case included dural suture with 4-0 thread (Figure 2D), return of the bone of the sellar floor and the application of artificial dura mater.

Neuronavigational guidance was used because of its ability to accurately determine location (Figure 1F), helping the operator resect the tumor and the eroded ethmoid sinus and anterior cranial base (Figure 2EF). The fat and muscle fascia of the quadriceps were obtained from the right leg. Along with the left mucoperiosteal flap of the nasal septum and inferior nasal meatus, the harvested leg tissues were used for anterior skull base reconstruction (Figure $2 \mathrm{H}$ ). 


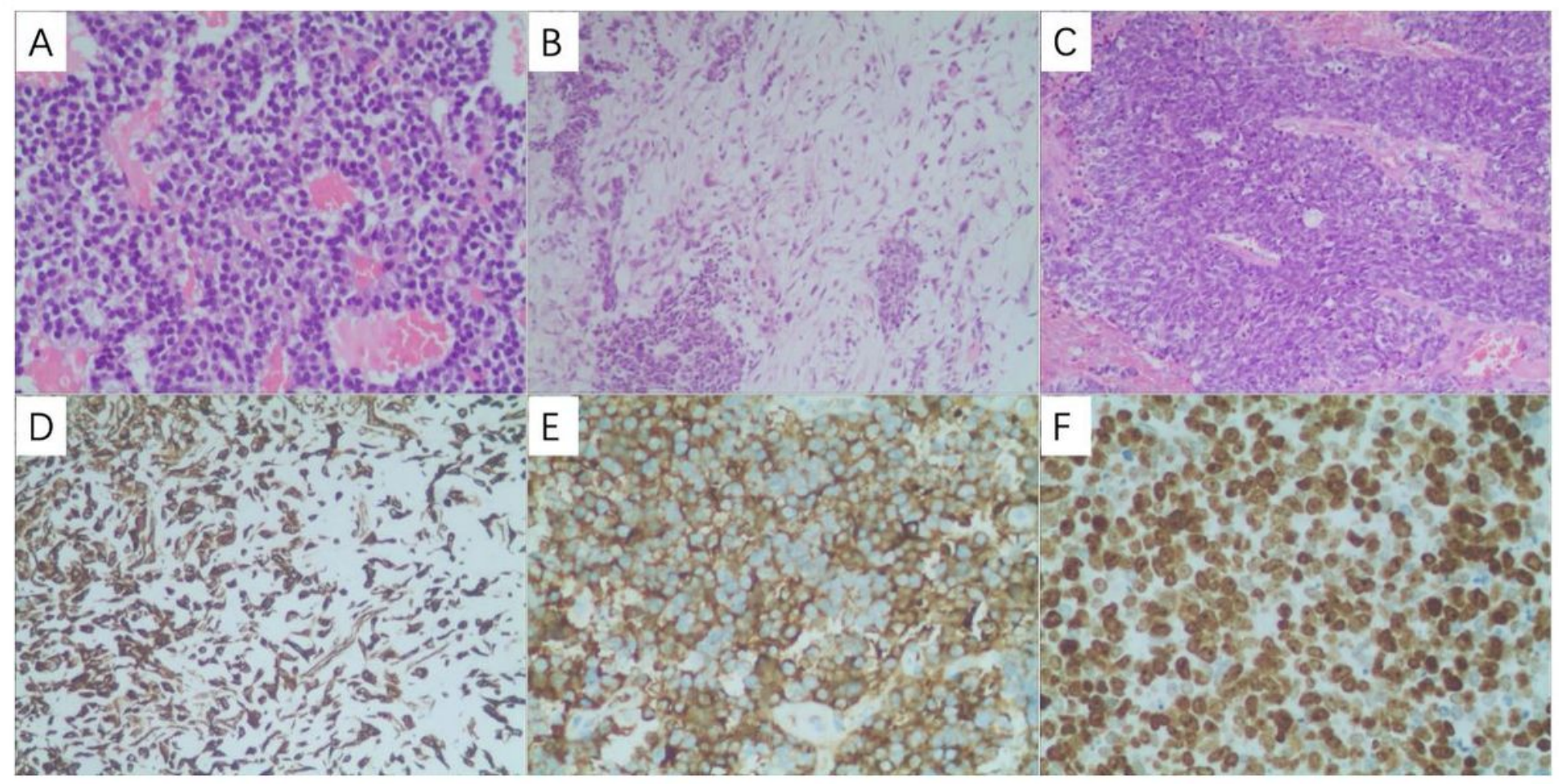

\section{Figure 3}

The immunohistochemical results (Figure 3) from the tumor involving the nasal cavity, paranasal sinuses and anterior cranial base were CK (+), EMA (-), GFAP (-), Ki-67 (more than 95\%), NSE (slightly positive), S100 (focally positive), Syn (focally positive), P63 (-), Olig - 2 (-), CD56 (+), CgA (focally positive), Des (focally positive), CK 8/18 (+), CK - L (+), CD99 (focally positive), Vim (focally positive), Myogenin (slightly positive), and MYOD1 (focally positive). 